\title{
Electrical Conductivity at the Core of a Magnetar
}

\author{
Sutapa Ghosh ${ }^{a) *}$, Sanchayita Ghosh $^{b)}$, Kanupriya Goswami ${ }^{c)}$, Somenath Chakrabarty ${ }^{a), d) \dagger}$ \\ and Ashok Goyal ${ }^{c), d) \ddagger}$ \\ a)Department of Physics, University of Kalyani, West Bengal 741 235, India \\ b)Department of Physics, Ranaghat College, West Bengal, India \\ c)Department of Physics and Astrophysics, University of Delhi, Delhi 110 007, India \\ d) IUCAA, Post bag 4, Ganeshkhind, Pune 411 007, India \\ PACS:05.20.Dd, 72.10.-d, 72.15.Lh, 79.60.Jd
}

\begin{abstract}
An expression for the electrical conductivity at the core of a magnetar is derived using Boltzmann kinetic equation with the relaxation time approximation. The rates for the relevant scattering processes, e.g., electron-electron and electron-proton are evaluated in presence of strong quantizing magnetic fields using tree level diagrams. It is found that in presence of a strong quantizing magnetic field, electrical conductivity behaves like a second rank tensor. However, if the zeroth Landau levels are only occupied by the charged particles, it again behaves like a scaler of a one dimensional system.
\end{abstract}

\footnotetext{
*E-mail:sutapa@klyuniv.ernet.in

${ }^{\dagger}$ E-mail:somenath@klyuniv.ernet.in

${ }^{\ddagger}$ E-mail:agoyal@ducos.ernet.in
} 


\section{INTRODUCTION}

With the recent observational discovery of magnetars, which are assumed to be strongly magnetized young neutron stars and also the possible sources of soft gamma-ray repeaters (SGR) and anomalous X-ray pulsars (AXP) [1], the study on the effect of strong magnetic fields on dense stellar matter has got a new dimension. In the recent years a lot of work have been done on the effect of strong magnetic field on the equation of state of dense stellar matter [5,6]. Some studies have also been done on the effect of strong magnetic field on elementary processes (e.g., weak and electromagnetic processes) occurring at the core region as well as at the envelope of strongly magnetized neutron stars [7,8]. During the past few years, in a number of publications we have reported our thorough investigations on the effect of strong magnetic field on the stability of dense quark matter and quark-hadron phase transition (both first order and second order) at the core of a strongly magnetized neutron star 9 [1]. We have also studied the effect of strong magnetic field on dense neutron matter with properly modifying the $\sigma-\omega-\rho$-meson type mean-field theory. In those work we have developed the relativistic version of mean field theory in presence of strong quantizing magnetic field using Hartree and Hartree-Fock model with $\sigma-\omega-\rho$ mesons exchange interaction [12,13].

In the recent years the transport coefficients in particular, the electrical conductivity of electrons at the crustal region of neutron stars have also been calculated in presence of strong magnetic fields [8]. Unfortunately, in none of these studies the dense core region of the star is taken into account. Now from the observational data of SGR and AXP, the strength of surface magnetic field of magnetars are predicted to be $\geq 10^{15} \mathrm{G}$. Then it is very easy to show by scalar Virial theorem that the magnetic field strength at the core region may go up to $10^{18} \mathrm{G}$. This is of course strong enough to affect most of the physical processes occurring at the core region. As a consequence, one of the most important physical phenomena, the transport properties of dense core matter, in particular, the electrical conductivity of electrons and transport properties of emitted neutrinos will be affected significantly by the 
strong magnetic field. To investigate the transport properties of dense matter at the core of a neutron star, we use the standard form of Boltzmann kinetic equation with relaxation time approximation. Since protons are too heavy compared to electrons, we have assumed that the electric current is solely due to the motion of electrons. Now the relaxation time is directly related to the rates of the relevant (weak and electromagnetic) processes. these processes are strongly affected by the presence of quantizing magnetic field. As a consequence, both qualitative and the quantitative nature of transport coefficients, e.g., electrical conductivity, viscosity and heat conductivity should change significantly in presence of strong magnetic

field. In a future publication we shall report the effect of strong magnetic field on the transport properties of neutrinos at the core region [14].

In the present article, we shall investigate the effect of strong magnetic field on the electrical conductivity at the core region of strongly magnetized neutron stars or magnetars. The paper is organized in the following manner: In section 2, we obtain the rates of the relevant processes in presence of strong magnetic field. In section 3, we shall derive an expression for the electrical conductivity from Boltzmann kinetic equation using relaxation time approximation. In the last section, we have given the conclusion and discussed the future perspective of the work.

\section{RATES}

To obtain electrical conductivity at the core of a magnetar, we have considered the following two electromagnetic processes relevant for electron transport: electron-electron scattering

$$
e\left(p_{1}\right)+e\left(p_{2}\right) \rightarrow e\left(k_{1}\right)+e\left(k_{2}\right)
$$

and electron-proton scattering

$$
e\left(p_{1}\right)+p\left(p_{2}\right) \rightarrow e\left(k_{1}\right)+p\left(k_{2}\right)
$$


where $p_{i}$ and $k_{i}$ are the initial and final four momenta. Since the rate of the electron-neutrino weak scattering, given by

$$
e+\nu_{e}\left(\bar{\nu}_{e}\right) \rightarrow e+\nu_{e}\left(\bar{\nu}_{e}\right)
$$

is several orders of magnitude less than the electromagnetic processes, it has almost no significance in the electrical conductivity calculation. Of course, this is one of the most important neutrino transport processes. For the same reason, we have neglected $e-e$ weak scattering.

To obtain electrical conductivity at the core of a magnetar, we first consider e-e scattering. For this process, both the direct as well as exchange diagrams are considered. From the definition, the transition matrix element is given by

$$
T_{f i}=-i \int j_{\mu}^{f i}(x) A^{\mu}(x) d^{4} x
$$

where $j_{\mu}^{f i}=e \bar{\psi}_{f} \gamma^{\mu} \psi_{i}$ is the electric current produced by the scattered electron and

$$
A^{\mu}(x)=-\int \frac{d^{4} q}{(2 \pi)^{4}} \frac{\exp \left[-i q\left(x-x^{\prime}\right)\right]}{q^{2}} j^{\mu}\left(x^{\prime}\right) d^{4} x^{\prime}
$$

is the electromagnetic field induced by the current $j^{\mu}\left(x^{\prime}\right)$ of the other electron and $q$ is the transferred four momentum.

Now in the case, when only the zeroth Landau levels are occupied by the electrons the positive energy Dirac spinor is given by

$$
\begin{aligned}
\psi^{(+1)}(x)= & \frac{1}{\left(L_{y} L_{z}\right)^{1 / 2}\left(\left(\varepsilon_{0}+m\right) \varepsilon_{0}\right)^{1 / 2}} \exp \left[-i\left(p_{0} t+p_{y} y+p_{z} z\right)\right] \\
& \left(\frac{e B}{\pi}\right)^{1 / 4} \exp \left[-\frac{1}{2} e B\left(x-\frac{p}{e B}\right)^{2}\right]\left(\begin{array}{c}
\varepsilon_{0}+m \\
0 \\
p_{z} \\
0
\end{array}\right)
\end{aligned}
$$

where $L_{y}$ and $L_{z}$ are the lengths along $y$ and $z$ directions, $\varepsilon_{0}=p_{0}=\left(p_{z}^{2}+m^{2}\right)^{1 / 2}$ is the modified form of single particle energy, $p_{y}$ and $p_{z}$ are $y$ and $z$ components of electron 
momentum, $B$ is the strength of magnetic field, assumed to be along positive $z$-direction (we have chosen the gauge $A^{\mu}=(0,0, x B, 0)$ ). The counter part of this spinor has no contribution when only the zeroth Landau level is occupied. Whereas, for low temperature case (the chemical potential $\mu>>T$, where $T$ is the temperature of the system. In the numerical calculation, we have taken $T=20 \mathrm{MeV}$ and found that the results are almost temperature independent for $5 \mathrm{MeV} \leq T \leq 30 \mathrm{MeV}$ ) the negative energy spinor solutions may be neglected.

Combining eqns.(4)-(6) and after integrating over $t^{\prime}, y^{\prime}, z^{\prime}$ (which result three $\delta$ functions), we have the transition matrix element for the direct $e-e$ scattering diagram

$$
\begin{aligned}
T_{f i}= & i \int \frac{d^{4} q d^{4} x}{2 \pi} \delta\left(q^{0}-p_{1}^{0}+k_{1}^{0}\right) \delta\left(q_{y}-p_{1 y}+k_{1 y}\right) \delta\left(q_{z}-p_{1 z}-k_{1 z}\right) \\
& \frac{\exp [i q x]}{q^{2}} \frac{\exp \left[-i q_{x} x^{\prime}\right]}{L_{y}^{2} L_{z}^{2}} \frac{e^{2}}{\left[16 p_{1}^{0} p_{2}^{0} k_{1}^{0} k_{2}^{0}\left(p_{1}^{0}+m\right)\left(k_{1}^{0}+m\right)\left(p_{2}^{0}+m\right)\left(k_{2}^{0}+m\right)\right]^{1 / 2}} \\
& \exp \left[-i\left\{\left(p_{2}^{0}-k_{2}^{0}\right) t-\left(p_{2 y}-k_{2 y}\right) y-\left(p_{2 z}-k_{2 z}\right) z\right\}\right]\left[J_{\mu}\left(x^{\prime}\right)\right]_{p_{1}, k_{1}}\left[J_{\mu}(x)\right]_{p_{2}, k_{2}} d x^{\prime}
\end{aligned}
$$

where

$$
\left[J_{\mu}(x)\right]_{p, k}=\bar{u}(k) \gamma^{\mu} u(p) I_{0 ; k_{y}, k_{z}}(x) I_{0 ; p_{y}, p_{z}}(x),
$$

$u$ and $\bar{u}$ are respectively the positive energy spinor and the corresponding adjoint and

$$
I_{0 ; k_{y}, k_{z}}=\left(\frac{e B}{\pi}\right)^{1 / 4} \exp \left[-\frac{e B}{2}\left(x-\frac{k_{y}}{e B}\right)^{2}\right]
$$

The integrals over $x$ and $x^{\prime}$ can very easily be evaluated by changing the variables to $r$ and $R$, given by $r=\left(x-x^{\prime}\right)$ and $R=\left(x+x^{\prime}\right) / 2$. The integration over $R$ the gives

$$
I_{R}=\left(\frac{\pi}{2 e B}\right)^{1 / 2} \exp \left[\frac{1}{2 e B}\left(p_{1 y}+p_{2 y}\right)^{2}\right]
$$

Similarly, the integration over $r$ is given by

$$
I_{r}=\left(\frac{\pi}{2 e B}\right)^{1 / 2} \exp \left[-2 e B X_{(d i r)}^{2}\right] \operatorname{Erfc}\left[-(2 e B)^{1 / 2} X_{(d i r)}\right]
$$

where $X_{(\text {dir })}=\left(k_{2 y}-p_{1 y}-K_{(d i r)}\right) / 2 e B, K_{(d i r)}^{2}=q_{y}^{2}+q_{z}^{2}-q_{0}^{2}$ and $\operatorname{Er} f c(x)$ is the complementary error function. After evaluating the integrals over $d^{4} q$ with the help of $\delta$-functions we have the transition matrix element for direct $e-e$ scattering 


$$
\begin{aligned}
T_{f i}^{(d i r)}= & -i \frac{e^{2}}{\left[16 p_{1}^{0} p_{2}^{0} k_{1}^{0} k_{2}^{0}\left(p_{1}^{0}+m\right)\left(p_{2}^{0}+m\right)\left(k_{1}^{0}+m\right)\left(k_{2}^{0}+m\right)\right]^{1 / 2}} \\
& {\left[J_{\mu}\left(p_{1}, k_{1}\right)\right]\left[J_{\mu}\left(p_{2}, k_{2}\right)\right] \frac{1}{4 L_{y}^{2} L_{z}^{2} e B} } \\
& \exp \left[\frac{1}{2 e B}\left(p_{1 y}+p_{2 y}\right)^{2}\right] \exp \left(-2 e B X_{(d i r)}^{2}\right) \\
& \operatorname{Erfc}\left(-(2 e B)^{1 / 2} X_{(d i r)}\right) \frac{1}{2 K_{(d i r)}}(2 \pi)^{3} \delta\left(p_{1}^{0}+p_{2}^{0}-k_{1}^{0}-k_{2}^{0}\right) \\
& \delta\left(p_{1 y}+p_{2 y}-k_{1 y}-k_{2 y}\right) \delta\left(p_{1 z}+p_{2 z}-k_{1 z}-k_{2 z}\right)
\end{aligned}
$$

Which may be written as

$$
T_{f i}^{(d i r)}=\Pi^{(d i r)}(2 \pi)^{3} \delta\left(p_{1}^{0}+p_{2}^{0}-k_{1}^{0}-k_{2}^{0}\right) \delta\left(p_{1 y}+p_{2 y}-k_{1 y}-k_{2 y}\right) \delta\left(p_{1 z}+p_{2 z}-k_{1 z}-k_{2 z}\right)
$$

The transition matrix element for $e-e$ exchange interaction can very easily be obtained from the direct one just by exchanging $k_{1}$ and $k_{2}\left(k_{1} \leftrightarrow k_{2}\right)$. Then we have

$$
\begin{aligned}
T_{f i}^{(e x)}= & -i \frac{e^{2}}{\left[16 p_{1}^{0} p_{2}^{0} k_{1}^{0} k_{2}^{0}\left(p_{1}^{0}+m\right)\left(p_{2}^{0}+m\right)\left(k_{1}^{0}+m\right)\left(k_{2}^{0}+m\right)\right]^{1 / 2}} \\
& {\left[J_{\mu}\left(p_{1}, k_{2}\right)\right]\left[J_{2}\left(p_{2}, k_{1}\right)\right] \frac{1}{4 L_{y}^{2} L_{z}^{2} e B} } \\
& \exp \left[\frac{1}{2 e B}\left(p_{1 y}+p_{2 y}\right)^{2}\right] \exp \left(-2 e B X_{(e x)}^{2}\right) \operatorname{Erf} c\left[-(2 e B)^{1 / 2} X_{e x)}\right] \frac{1}{2 K_{(e x)}}(2 \pi)^{3} \\
& \delta\left(p_{1}^{0}+p_{2}^{0}-k_{1}^{0}-k_{2}^{0}\right) \delta\left(p_{1 y}+p_{2 y}-k_{1 y}-k_{2 y}\right) \delta\left(p_{1 z}+p_{2 z}-k_{1 z}-k_{2 z}\right)
\end{aligned}
$$

where $X_{(e x)}=\left(k_{1 y}-p_{1 y}-K_{(e x)}\right) / 2 e B$ and $K_{(e x)}=K_{(d i r)}$. Which may also be written in the form

$$
\begin{aligned}
T_{f i}^{(e x)}= & \Pi^{(e x)}(2 \pi)^{3} \delta\left(p_{1}^{0}+p_{2}^{0}-k_{1}^{0}-k_{2}^{0}\right) \delta\left(p_{1 y}+p_{2 y}-k_{1 y}-k_{2 y}\right) \\
& \delta\left(p_{1 z}+p_{2 z}-k_{1 z}-k_{2 z}\right)
\end{aligned}
$$

Now from the elementary definition of rate per unit volume for the scattering process, we have the differential rate for $e-e$ scattering

$$
\begin{aligned}
d W= & \left|\Pi^{(d i r)}+\Pi^{(e x)}\right|^{2}(2 \pi)^{3} \delta\left(p_{1}^{0}+p_{2}^{0}-k_{1}^{0}-k_{2}^{0}\right) \delta\left(p_{1 y}+p_{2 y}-k_{1 y}-k_{2 y}\right) \delta\left(p_{1 z}+p_{2 z}-k_{1 z}-k_{2 z}\right) \\
& \frac{d k_{1 y} d k_{1 z} d k_{2 y} d k_{2 z} d p_{2 y} d p_{2 z}}{(2 \pi)^{6}}
\end{aligned}
$$


Hence to obtain the differential rate $d W$ of the processes we have to calculate $\left|\Pi^{(\text {dir })}\right|^{2}$, $\left|\Pi^{(e x)}\right|^{2}$ and the cross term. Further, to compute these quantities, we have to evaluate the traces of the products of $\gamma$-matrices. Using the standard techniques, we have computed the traces of the products of $\gamma$-matrices. Then we have

$$
\begin{aligned}
d W_{(d i r)}= & \frac{e^{4}}{p_{1}^{0} p_{2}^{0} k_{1}^{0} k_{2}^{0}} \exp \left[\frac{1}{e B}\left(p_{1 y}+p_{2 y}\right)^{2}\right] \exp \left(-4 e B X_{(d i r)}^{2}\right) \\
& \left\{\operatorname{Erfc}\left[-(2 e B)^{1 / 2} X_{(d i r)}\right]\right\}^{2}\left[\left(p_{1} \cdot p_{2}\right)\left(k_{1} \cdot k_{2}\right)+\left(p_{1} \cdot k_{2}\right)\left(k_{1} \cdot p_{2}\right)-m^{2}\left(p_{2} \cdot k_{2}\right)-m^{2}\left(p_{1}, k_{1}\right)\right. \\
& \left.+2 m^{4}\right] \frac{1}{8 \pi^{2} K_{(d i r)}^{2}} \frac{1}{(2 \pi)^{3}} \delta\left(p_{1}^{0}+p_{2}^{0}-k_{1}^{0}-k_{2}^{0}\right) \delta\left(p_{1 y}+p_{2 y}-k_{1 y}-k_{2 y}\right) \\
& \delta\left(p_{1 z}+p_{2 z}-k_{1 z}-k_{2 z}\right) d k_{1 y} d k_{1 z} d k_{2 y} d k_{2 z} d p_{2 y} d p_{2 z}
\end{aligned}
$$

Similarly, the exchange term is obtained by $k_{1} \leftrightarrow k_{2}$ and is given by

$$
\begin{aligned}
d W_{(e x)}= & \frac{e^{4}}{p_{1}^{0} p_{2}^{0} k_{1}^{0} k_{2}^{0}} \exp \left[\frac{1}{e B}\left(p_{1 y}+p_{2 y}\right)^{2}\right] \exp \left(-4 e B X_{(e x)}^{2}\right) \operatorname{Erfc}\left[-(2 e B)^{1 / 2} X_{(e x)}\right]^{2} \\
& {\left[\left(p_{1} \cdot p_{2}\right)\left(k_{1} \cdot k_{2}\right)+\left(p_{1} \cdot k_{1}\right)\left(p_{2} \cdot k_{2}\right)-m^{2}\left(p_{2} \cdot k_{1}\right)-m^{2}\left(p_{1} \cdot k_{2}\right)+2 m^{4}\right] \frac{1}{8 \pi^{2} K_{(e x)}^{2}} } \\
& \frac{1}{(2 \pi)^{3}} \delta\left(p_{1}^{0}+p_{2}^{0}-k_{1}^{0}-k_{2}^{0}\right) \delta\left(p_{1 y}+p_{2 y}-k_{1 y}-k_{2 y}\right) \delta\left(p_{1 z}+p_{2 z}-k_{1 z}-k_{2 z}\right) \\
& d k_{1 y} d k_{1 z} d k_{2 y} d k_{2 z} d p_{2 y} d p_{2 z}
\end{aligned}
$$

Finally the cross term whose evaluation is a bit lengthy, but straight-forward, is given by

$$
\begin{aligned}
d W_{(\text {cross })}= & \frac{e^{4}}{p_{1}^{0} p_{2}^{0} k_{1}^{0} k_{2}^{0}} \exp \left[\frac{1}{e B}\left(p_{1 y}+p_{2 y}\right)^{2}\right] \exp \left[-2 e B\left(X_{(d i r)}^{2}+X_{(e x)}^{2}\right)\right] \\
& \operatorname{Erfc}\left[-(2 e B)^{1 / 2} X_{(d i r)}\right] \operatorname{Erfc}\left[-(2 e B)^{1 / 2} X_{(e x)}\right] \\
& {\left[-2\left(k_{1} \cdot k_{2}\right)\left(p_{1} \cdot p_{2}\right)+m^{2}\left(p_{2} \cdot k_{2}\right)+m^{2}\left(p_{2} \cdot k_{1}\right)+m^{2}\left(p_{2} \cdot p_{1}\right)+\right.} \\
& \left.m^{2}\left(p_{1} \cdot k_{1}\right)+m^{2}\left(p_{1} \cdot k_{2}\right)+m^{2}\left(k_{1} \cdot k_{2}\right)-2 m^{4}\right] \\
& \frac{1}{8 \pi^{2} K_{(d i r)} K_{(e x)}} \frac{1}{\left(2 \pi^{3}\right)} \delta\left(p_{1}^{0}+p_{2}^{0}-k_{1}^{0}-k_{2}^{0}\right) \delta\left(p_{1 y}+p_{2 y}-k_{1 y}-k_{2 y}\right) \delta\left(p_{1 z}+p_{2 z}-k_{1 z}-k_{2 z}\right) \\
& d k_{1 y} d k_{1 z} d k_{2 y} d k_{2 z} d p_{2 y} d p_{2 z}
\end{aligned}
$$

The simplified form of these rates can very easily be obtained by integrating over the momentum components $k_{1 z}, k_{2 z}, p_{2 y}$ and $p_{2 z}$ with the help of $\delta$-functions. Then we have after some simple algebraic manipulation 


$$
\begin{aligned}
d W_{(d i r)}= & \frac{e^{4}}{p_{1}^{0} k_{1}^{0} p_{2}^{0} k_{2}^{0}} \exp \left[\frac{1}{e B}\left(k_{1 y}+k_{2 y}\right)^{2}\right] \exp \left[\frac{1}{e B}\left(k_{2 y}-k_{1 y}-K_{(d i r)}\right)\right] \\
& {\left[\operatorname{Erfc}\left\{-\frac{1}{(2 e B)^{1 / 2}}\left(k_{2 y}-k_{1 y}-K_{(d i r)}\right)\right\}\right]^{2}\left[\left(p_{1}^{0} p_{2}^{0}-p_{1 z} p_{2 z}\right)\left(k_{1}^{0} k_{2}^{0}-k_{1 z} k_{2 z}\right)+\right.} \\
& \left.\left(p_{1}^{0} k_{2}^{0}-p_{1 z} k_{2 z}\right)\left(k_{1}^{0} p_{2}^{0}-k_{1 z} p_{2 z}\right)-m^{2}\left(p_{2}^{0} k_{2}^{0}-p_{2 z} k_{2 z}\right)-m^{2}\left(p_{1}^{0} k_{1}^{0}-p_{1 z} k_{1 z}\right)+2 m^{4}\right] \\
& \left.\frac{1}{8 \pi^{2} K_{(d i r)}^{2}} \frac{1}{(2 \pi)^{3}} d k_{1 y} d k_{2 y}\right|_{k_{1 z}=p_{F}, k_{2 z}=k_{2 z}^{(R)}, p_{2 z}=p_{1 z}+k_{1 z}-k_{2 z}} \\
& \frac{p_{F}}{\left|f^{\prime}\left(k_{2 z}\right)\right|_{k_{2 z}=k_{2 z}^{(R)}}}
\end{aligned}
$$

where $K_{(d i r)}^{2}=\left(p_{1 z}-p_{F}\right)^{2}-\left(p_{1}^{0}-\mu\right)^{2}, \mu$ and $p_{F}$ are the electron chemical potential and Fermi momentum respectively,

$$
k_{2 z}^{(R)}=\frac{m\left(p_{1}^{0}+\mu\right)}{\left[2 m^{2}+2 p_{1}^{0} \mu-2 p_{1 z} p_{F}\right]^{1 / 2}}
$$

is the root of the transcendental equation $p_{1}^{0}+p_{2}^{0}-k_{1}^{0}-k_{2}^{0}=0$ and

$$
\left|f^{\prime}\left(k_{2 z}\right)\right|_{k_{2 z}=k_{2 z}^{(R)}}=\left|\frac{\left(b-k_{2 z}^{(R)}\right)}{\left\{\left(b-k_{2 z}^{(R)}\right)^{2}+m^{2}\right\}^{1 / 2}}-\frac{k_{2 z}^{(R)}}{\left(k_{2 z}^{(R) 2}+m^{2}\right)^{1 / 2}}\right|
$$

where $b=p_{1 z}+p_{F}$

Similarly, for the exchange interaction we have

$$
\begin{aligned}
d W_{(e x)}= & \frac{e^{4}}{p_{1}^{0} k_{1}^{0} p_{2}^{0} k_{2}^{0}} \exp \left[\frac{1}{e B}\left(k_{1 y}+k_{2 y}\right)^{2}\right] \exp \left[-\frac{1}{e B}\left(k_{1 y}-k_{2 y}-K_{(e x)}\right)^{2}\right] \\
& \left\{\operatorname{Erfc}\left[-\frac{k_{1 y}-k_{2 y}-K_{(e x)}}{(2 e B)^{1 / 2}}\right]\right\}^{2}\left[\left(p_{1} \cdot p_{2}\right)\left(k_{1} \cdot k_{2}\right)+\left(p_{1} \cdot k_{1}\right)\left(p_{2} \cdot k_{2}\right)\right. \\
& \left.-m^{2}\left(p_{2} . k_{1}\right)-m^{2}\left(p_{1} \cdot k_{2}\right)+2 m^{4}\right] \frac{1}{8 \pi^{2} K_{(e x)}^{2}} \frac{1}{(2 \pi)^{3}} \\
& \left.d k_{1 y} d k_{2 y}\right|_{k_{2 z}=k_{F}, k_{1 z}=k_{1 z}^{(R)}, k_{2 z}=k_{1 z}+p_{1 z}-k_{2 z}} \frac{p_{F}}{\left|f^{\prime}\left(k_{2 z}\right)\right|_{k_{2 z}=k_{2 z}^{(R)}}^{(R)}}
\end{aligned}
$$

where $K_{(e x)}^{2}=\left(p_{1 z}-p_{F}\right)^{2}-\left(p_{1}^{0}-\mu\right)^{2}$. Finally for the mixed term, we have

$$
\begin{aligned}
d W_{(\text {cross })}= & \frac{e^{4}}{p_{1}^{0} k_{1}^{0} p_{2}^{0} k_{2}^{0}} \exp \left[\frac{1}{e B}\left(k_{1 y}+k_{2 y}\right)^{2}\right] \exp \left[-\frac{1}{2 e B}\left(k_{2 y}-k_{1 y}-K_{(d i r)}\right)^{2}\right] \\
& \exp \left[-\frac{1}{2 e B}\left(k_{1 y}-k_{2 y}-K_{(e x)}\right)^{2}\right] \operatorname{Erfc}\left[-\frac{k_{2 y}-k_{1 y}-K_{(d i r)}}{(2 e B)^{1 / 2}}\right] \\
& \operatorname{Erfc}\left[-\frac{k_{1 y}-k_{2 y}-K_{(e x)}}{(2 e B)^{1 / 2}}\right]\left[-2\left(p_{1} \cdot p_{2}\right)\left(k_{1} \cdot k_{2}\right)+m^{2}\left(p_{2} \cdot k_{2}\right)+m^{2}\left(p_{2} \cdot k_{1}\right)+\right. \\
& \left.m^{2}\left(p_{1} \cdot p_{2}\right)+m^{2}\left(p_{1} \cdot k_{1}\right)+m^{2}\left(p_{1} \cdot k_{2}\right)+m^{2}\left(k_{1} \cdot k_{2}\right)-2 m^{4}\right]
\end{aligned}
$$




$$
\begin{aligned}
& \frac{1}{8 \pi^{2} K_{(d i r)} K_{(e x)}} \frac{1}{(2 \pi)^{3}} \\
& \left.d k_{1 y} d k_{2 y}\right|_{k_{2 z}=k_{F}, k_{1 z}=k_{1 z}^{(R)}, k_{2 z}=k_{1 z}+p_{1 z}-k_{2 z}} \frac{p_{F}}{\left|f^{\prime}\left(k_{1 z}\right)\right|_{k_{1 z}=k_{1 z}^{(R)}}}
\end{aligned}
$$

In the case of $e-p$ scattering, given by eqn.(2) the direct diagram only contributes and the form of trace term will be slightly different from that of $e-e$ direct term, given by

$$
\begin{aligned}
& 32\left(p_{1}^{0}+m\right)\left(k_{1}^{0}+m\right)\left(p_{2}^{0}+M^{*}\right)\left(k_{2}^{0}+M^{*}\right)\left[\left(p_{1} \cdot p_{2}\right)\left(k_{1} \cdot k_{2}\right)+\right. \\
& \left.\left(p_{1} \cdot k_{2}\right)\left(k_{1} \cdot p_{2}\right)-m^{2}\left(p_{2}, k_{2}\right)-M^{* 2}\left(p_{1} \cdot k_{1}\right)+2 m^{2} M^{* 2}\right]
\end{aligned}
$$

where $M^{*}$ is the effective proton mass in the Hartree type mean field model in presence of strong magnetic field [12]. In this case we have to use $p_{2}^{0}=\left(p_{2 z}^{2}+M^{2}\right)^{1 / 2}$ and $k_{2}^{0}=$ $\left(k_{2 z}^{2}+M^{2}\right)^{1 / 2}$ and make necessary changes in eqn.(20) of $d W_{(d i r)}$ for $e-e$ scattering to

obtain $d W_{(d i r)}^{(e p)}$. In the next section we shall use these differential rates (eqns.(20), (23), (24) and $\left.d W_{(d i r)}^{(e p)}\right)$ to obtain the electrical conductivity.

\section{ELECTRICAL CONDUCTIVITY}

To compute the electrical conductivity of the magnetized stellar matter, we consider the simplest form of Boltzmann kinetic equation for electrons which are assumed to be slightly out of local thermodynamic equilibrium. The kinetic equation is then given by [15, 16

$$
\frac{\partial f}{\partial t}+\frac{p_{i}}{p_{0}} \nabla_{i} f+\dot{p}_{i} \frac{\partial f}{\partial p_{i}}=C
$$

Where $f$ is the non-equilibrium distribution function and $C$ is the collision term, given by

$$
\begin{aligned}
C= & \int \frac{d^{3} p_{2}}{(2 \pi)^{3}} \frac{d^{3} p_{3}}{(2 \pi)^{3}} \frac{d^{3} p_{4}}{(2 \pi)^{3}}(2 \pi)^{3} \delta\left(p_{1}^{0}+p_{2}^{0}-k_{1}^{0}-k_{2}^{0}\right) \\
& \delta\left(p_{1 y}+p_{2 y}-k_{1 y}-k_{2 y}\right) \delta\left(p_{1 z}+p_{2 z}-k_{1 z}-k_{2 z}\right) \\
& \left|T_{f i}\right|^{2} \mathcal{F}
\end{aligned}
$$

where $\mathcal{F}=\left[f_{3} f_{4}\left(1-f_{1}\right)\left(1-f_{2}\right)-f_{1} f_{2}\left(1-f_{3}\right)\left(1-f_{4}\right)\right]$, and $\left(1-f_{i}\right)$ 's are the Pauli blocking factors. Replacing collision term by the relaxation time approximation, we have the relevant portion of the kinetic equation needed to obtain the electrical conductivity. 


$$
\dot{p}_{i} \frac{\partial f}{\partial p_{i}}=-\frac{\left(f-f_{0}\right)}{\tau}
$$

where $f_{0}$ is the local equilibrium distribution function for electrons (Fermi distribution), given by

$$
f_{0}=\frac{1}{\exp \left(\beta(x)\left(\left(p_{z}^{2}+p_{\perp}^{2}+m^{2}\right)^{1 / 2}-\mu(x)\right)\right)+1}
$$

Here $p_{z}$ is the longitudinal momentum and $p_{\perp}=(2 \nu e B)^{(1 / 2)}$ is the corresponding transverse part for the electrons, $T(x)=1 / \beta(x)$ and $\mu(x)$ are the local temperature and chemical potential. When only the zeroth Landau levels $(\nu=0)$ are occupied by the electrons, the transverse momentum becomes exactly zero.

We now consider the first order approximation, i.e., the system is very close to its local equilibrium configuration, then we can write $f(p, x)=f_{0}(p)+\delta f(p, x)$ where $\delta f$ is a measure of its deviation from local statistical equilibrium configuration. Then we have $\mathcal{F}=-(\delta f)\left\{f_{3,0} f_{4,0}\left(1-f_{2,0}\right)-f_{2,0}\left(1-f_{3,0}\right)\left(1-f_{4,0}\right)\right\}$, where $f_{i, 0}$ 's are the equilibrium distribution function for the $i$ th component (Fermi distribution). The we can write

$$
\frac{\partial f_{0}}{\partial p_{i}}=-f_{0}\left(1-f_{0}\right) \beta \frac{p_{i}}{\varepsilon}
$$

Expressing the force term in the form, $\dot{p}_{i}=e E_{i}$ we have after using eqn.(3)

$$
\frac{\delta f}{\tau}=f_{0}\left(1-f_{0}\right) \beta q E_{i} \frac{p_{i}}{\varepsilon}
$$

Then just by inspection, it is very easy to realize that the relaxation time is obtained by evaluating the integrals over $d k_{1 y}$ and $d k_{2 y}$ in the expressions for the rates and the relation with the total rate of the processes is given by

$$
\tau\left(p_{1 z}\right)=\frac{1}{W}
$$

where $W=W_{(d i r)}^{(e e)}+W_{(e x)}^{(e e)}+W_{(c r o s s)}^{(e e)}+W_{(d i r)}^{(e p)}$ therefore,

$$
\frac{1}{\tau}=\frac{1}{\tau_{(d i r)}^{(e e)}}+\frac{1}{\tau_{(e x)}^{(e e)}}+\frac{1}{\tau_{(m i x)}^{(e e)}}+\frac{1}{\tau_{(d i r)}^{(e p)}}
$$


the well know Matthiessen's rule [17]. Now the expression for electric current is given by

$$
j_{i}=e \int f(x, p) \frac{p_{i}}{\varepsilon} \frac{d^{3} p}{(2 \pi)^{3}}
$$

Because of randomness in the system, the local current vanishes at equilibrium. Then the nonzero part of electric current is obtained by using the first order deviation $\delta f(p, x)$ and is given by

$$
j_{i}=\frac{e^{2} \beta}{(2 \pi)^{3}} \int \frac{p_{i}}{\varepsilon} \tau f_{0}\left(1-f_{0}\right) \frac{p_{j}}{\varepsilon} d^{3} p E_{j}
$$

In presence of a strong magnetic field of strength $B>B_{c}^{(e)}$, the quantum limit of magnetic field, where $B_{c}^{(e)}=4.4 \times 10^{13} \mathrm{G}$, we have $d^{3} p=2 \pi e B d p_{z}$. Then writing Ohm's law in the form $j_{i}=\sigma_{i j} E_{j}$, we have

$$
\sigma_{i j}=\frac{e^{2} \beta e B}{2 \pi^{2}} \int \tau f_{0}\left(1-f_{0}\right) \frac{p_{i} p_{j}}{\varepsilon^{2}} d p_{z}
$$

Which is second rank tensor with the components $\sigma_{z z}, \sigma_{z \perp}, \sigma_{\perp z}$ and $\sigma_{\perp \perp}$. Since we are interested for $\nu=0$ case only, i.e., when the lowest Landau levels are populated, we have $\sigma_{\perp \perp}=\sigma_{\perp z}=\sigma_{z \perp}=0$ and

$$
\sigma_{z z}=\frac{e^{2} \beta}{2 \pi^{2}} e B \int \tau\left(p_{z}\right) f_{0}\left(1-f_{0}\right) \frac{p_{z}^{2}}{\varepsilon^{2}} d p_{z}
$$

which is the only non-zero component. The electron transport in this special case becomes essentially one dimensional- along the direction of magnetic field.

In fig.(1) we have plotted the variation of $\sigma_{z z}$ with electron density for (a) $B=10^{3} \times B_{c}^{(e)}$, (b) $B=5 \times 10^{3} \times B_{c}^{(e)},(\mathrm{c}) B=10^{4} \times B_{c}^{(e)},(\mathrm{d}) B=5 \times 10^{4} \times B_{c}^{(e)}$ and $(\mathrm{e}) B=10^{5} \times B_{c}^{(e)}$, where $B_{c}^{(e)} \approx 4.4 \times 10^{13} \mathrm{G}$, the quantum limit for electrons. In fig.(2) we have shown the variation of the nonzero component of electrical conductivity $\sigma_{z z}$ with magnetic field strength for (a) $n_{e}=n_{e}^{0}$, (b) $n_{e}=10 n_{e}^{0}$, (c)ne $=50 n_{e}^{0}$, and (d) $n_{e}=100 n_{e}^{0}$, where $n_{e}^{0}=10^{-2} \mathrm{fm}^{-3}$, the typical electron density in a non-magnetic neutron star. In both these cases we have not taken $\beta$ equilibrium condition into account. The density of electron is in some sense arbitrary. Therefore, we now consider an interacting $n-p-e$ system in $\beta$-equilibrium, which is the 
real physical picture at the core region of a neutron star. Then we have $n_{p}=n_{e}$, the charge neutrality condition, $\mu_{n}=\mu_{p}+\mu_{e}$, the $\beta$-equilibrium condition (we assume that the neutrinos are non-degenerate, leave the system as soon as they are produced) and baryon number density $n_{B}=n_{p}+n_{n}$ remains invariant in electromagnetic and weak interaction processes. Solving these constraints self-consistently at the core of a magnetar, we obtain the chemical potential of the constituents and hence the density of electrons for various baryon densities and magnetic field strengths. We have assumed a fixed temperature $T=20 \mathrm{MeV}$. In fig.(3) we have shown the variation of $\sigma_{z z}$ with the strength of magnetic field for a fixed baryon number density (in the numerical computation we have taken $n_{B}=4 n_{0}, n_{0}=0.17 \mathrm{fm}^{-3}$ ) assuming that the system is in $\beta$-equilibrium. In fig.(4) we have plotted $\sigma_{z z}$ against the electron density for a fixed magnetic field strength $\left(B=10^{5} B_{c}^{(e)}\right)$ in $\beta$-equilibrium condition.

\section{CONCLUSION}

We have studied the electrical conductivity at the core of a magnetar using Boltzmann kinetic equation with the relaxation time approximation. The latter is obtained from the rates of the electromagnetic processes, which essentially control the electron transport in the medium. We have further noticed that the electrical conductivity behaves like a second rank tensor above the critical value (quantum limit) of magnetic field strength. However, only $\sigma_{z z}$ components is non-zero when the lowest Landau levels are occupied by the charged particles. The system effectively becomes one-dimensional in presence of quantizing magnetic field- electrons can move along the field direction. The electric current vanishes in the plane transverse to the direction of magnetic field. Since it is impossible to compute the rates analytically (even it is very difficult to obtain numerically) with the non-zero values of Landau quantum numbers for all the four charged particles, we have assumed that only the zeroth Landau levels are occupied. This makes our like much simpler. The assumption of only zero Landau quantum number is also justified by the presence of intense magnetic field at the core region of magnetars. Fig.(1) shows that there exist cut off densities, beyond which, 
the Pauli blocking factor supresses the electron scattering and as a result the conductivity becomes zero. This figure also shows that the cut off density increases with the increas of magnetic field strength. As the magnetic field increases, for a given baryon number density, the chemical potential of electrons decreases, which further reduces the Pauli suppression factor. Similarly, we have noticed from fig.(2) that there is a minimum value for magnetic field strength for a particular baryon number density below which the conductivity again becomes zero because of same reason as discussed above. On the other hand, in the case of $\beta$-equilibrium condition, electrons are generated self-consistently. So there are no such cut off values for the density or magnetic field and the variation is smooth.

With this simplified picture, we have obtained the rates and finally $\sigma_{z z}$ for various baryon densities and magnetic field strengths. 


\section{FIGURES}

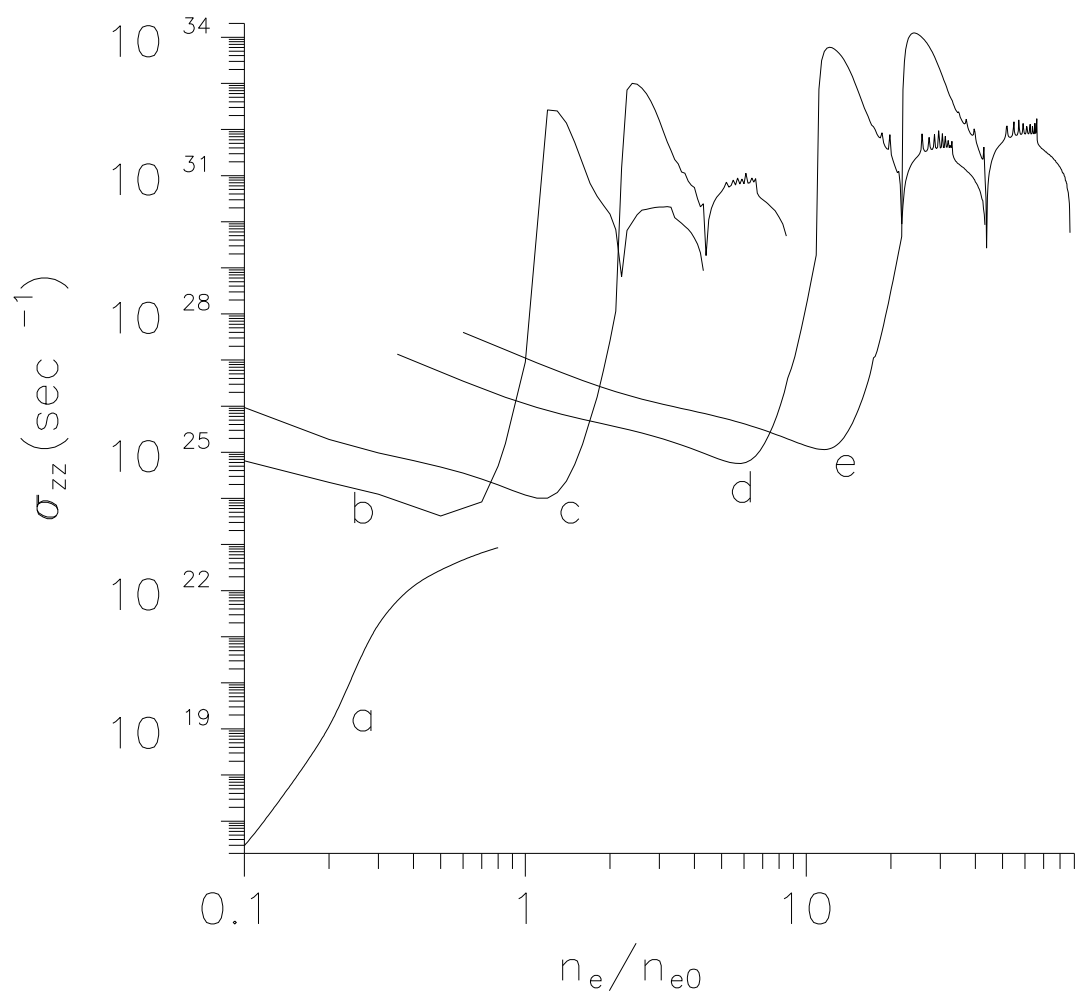

FIG. 1. Variation of $\sigma_{z z}$ in $\sec ^{-1}$ with electron density for (a) $B=10^{3} \times B_{c}^{(e)}$, (b) $B=5 \times 10^{3} \times B_{c}^{(e)},(\mathrm{c}) B=10^{4} \times B_{c}^{(e)},(\mathrm{d}) B=5 \times 10^{4} \times B_{c}^{(e)}$ and $(\mathrm{e}) B=10^{5} \times B_{c}^{(e)}$.

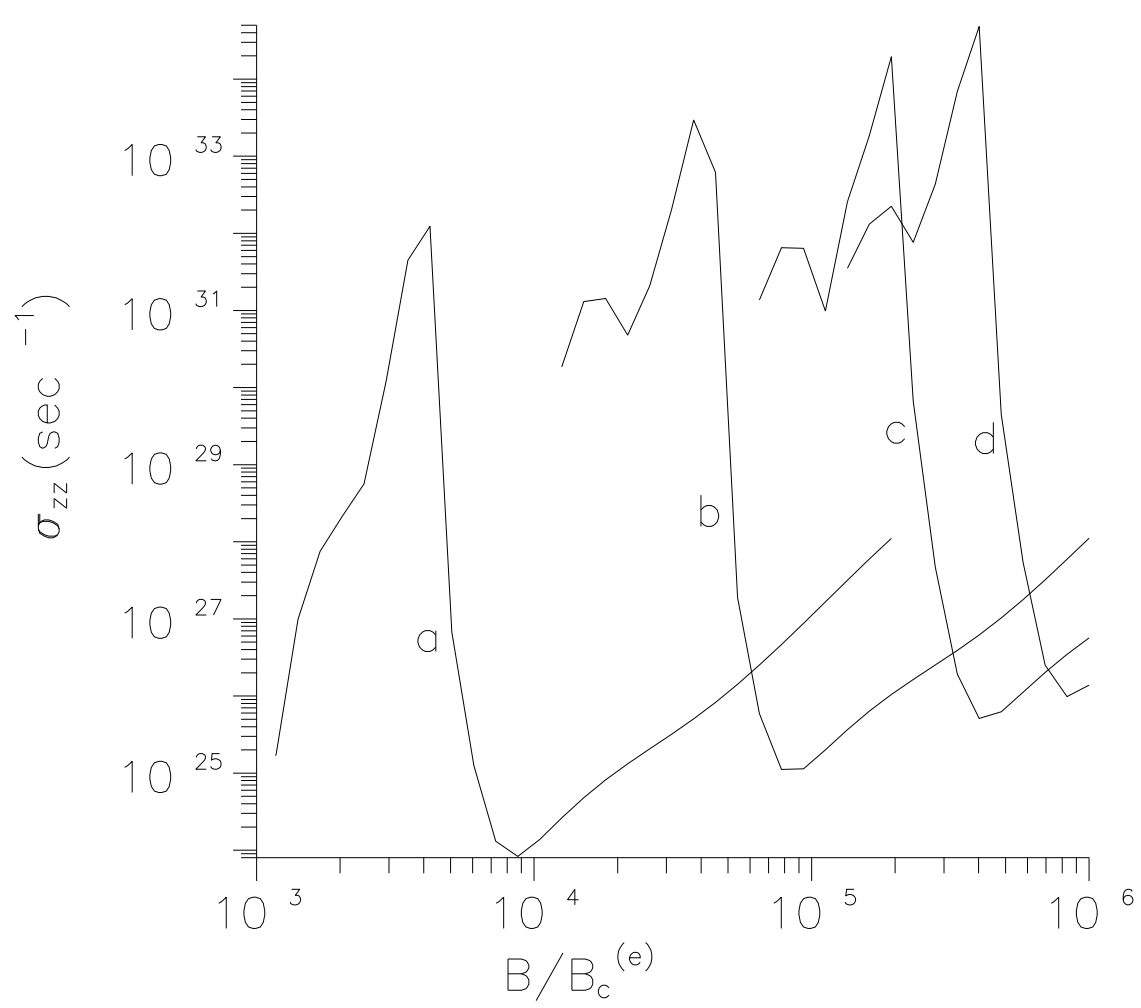


FIG. 2. Variation of $\sigma_{z z}$ in $\sec ^{-1}$ with magnetic field strength, for $(\mathrm{a}) n_{e}=n_{e}^{0}$, (b) $n_{e}=10 n_{e}^{0},(\mathrm{c}) n_{e}=50 n_{e}^{0}$ and $(\mathrm{d}) n_{e}=100 n_{e}^{0}$.

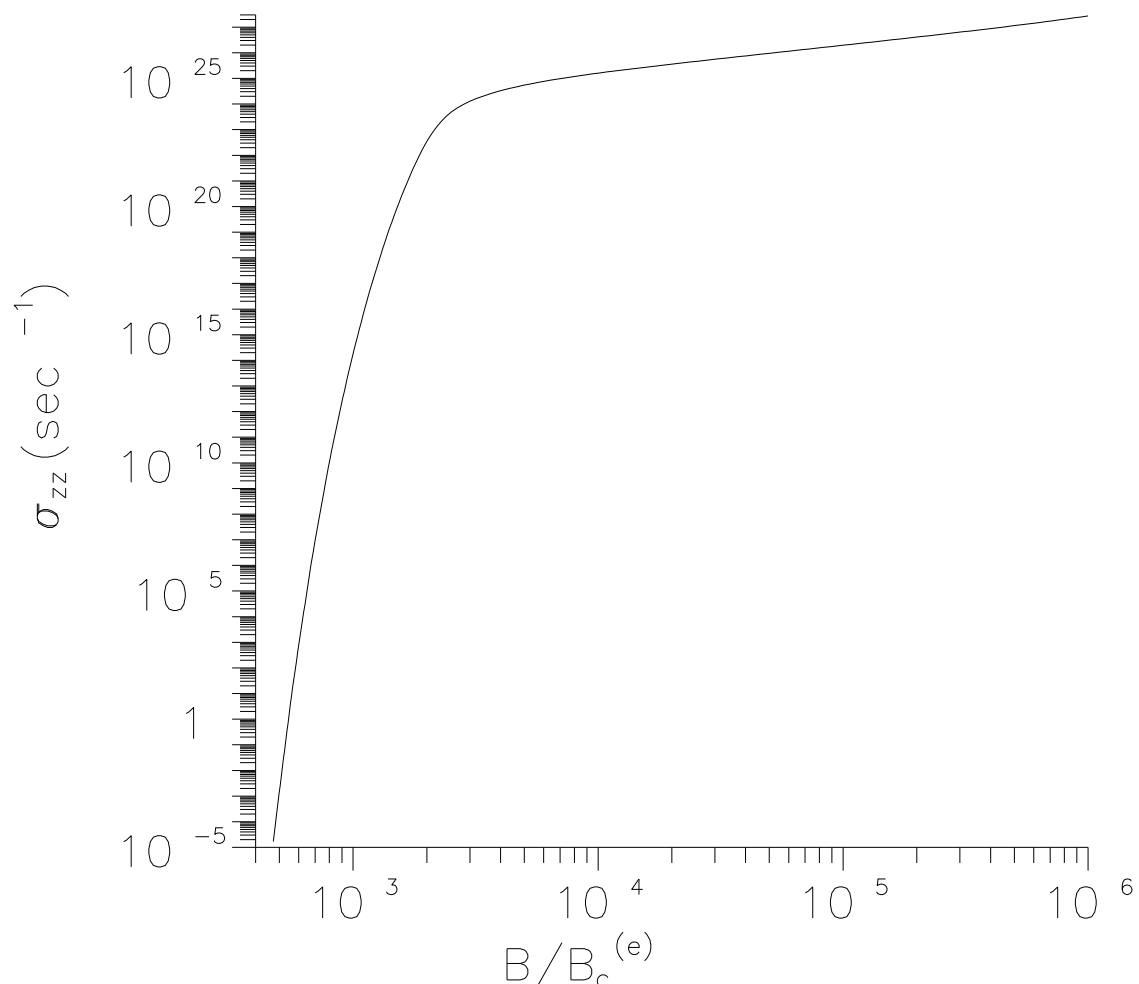

FIG. 3. Variation of $\sigma_{z z}$ with magnetic field strength for fixed baryon density $\left(n_{B}=4 n_{0}\right)$. The system is assumed to be in $\beta$-equilibrium. 


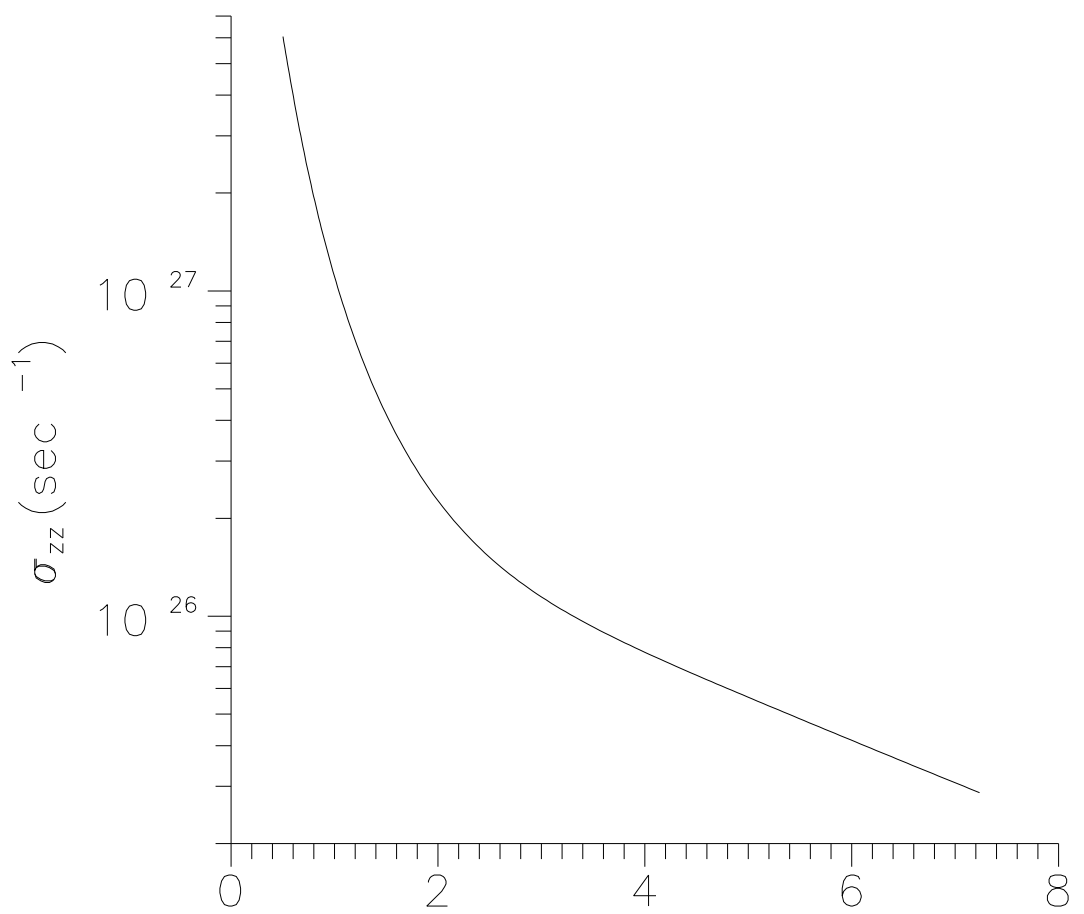

FIG. 4. Variation of $\sigma_{z z}$ with electron density for constant magnetic field strength $\left(B=10^{5} \times B_{c}^{(e)}\right)$. The system is assumed to be in $\beta$-equilibrium. 


\section{REFERENCES}

[1] C. Kouveliotou, et al., Nature 393, 235 (1998).

[2] K. Hurley, et al., Astrophys. Jour. 442, L111 (1999).

[3] S. Mereghetti and L. Stella, Astrophys. Jour. 442, L17 (1999).

[4] J. van Paradihs, R.E. Taam and E.P.J. van den Heuvel, Astron. Astrophys. 299, L41 (1995); S. Mereghetti, astro-ph/99111252; see also A. Reisenegger, astro-ph/01003010.

[5] C.Y. Cardall, M. Prakash and J.M. Lattimer, astro-ph/0011148.

[6] In-Saeng Suh and G.J. Mathews, astro-ph/9912301.

[7] F. Özel, astro-ph/0103227; T. Ghosh, Ph.D. Thesis, Univ. of Kalyani (unpublished).

[8] A.Y. Potekhin and D.G. Yakovlev, astro-ph/0105261 and references therein.

[9] S. Chakrabarty, Astrophys. Space Sci. 213, 121 (1994); S. Chakrabarty and A. Goyal, Mod. Phys. Lett. A9, 3611 (1994); S. Chakrabarty, Phys. Rev. D51, 4591 (1995).

[10] S. Chakrabarty and P. K. Sahu, Phys. Rev. D54, 1306 (1996); S. Chakrabarty, D. Bandopadhyay and S. Pal, Int. Jour. Mod. Phys. A13, 295 (1998); D. Bandopadhyaya, S. Chakrabarty, P. Dey and S. Pal, Phys. Rev. D58, 121301 (1998).

[11] T. Ghosh and S. Chakrabarty, Phys. Rev. D63, 0403006 (2001); T. Ghosh and S. Chakrabarty, Int. Jour. Mod. Phys. D10, 89 (2001).

[12] S. Chakrabarty, D. Bandopadhyay and S. Pal, Phys. Rev. Lett. 78, 2898 (1997).

[13] D. Bandopadhyay, S. Chakrabarty and S. Pal, Phys. Rev. Lett. 79, 2176 (1997).

[14] K. Huang, Statistical Mechanics, 2nd. ed., John Wiley and Sons, New York (1987).

[15] E.M. Lifshitz and L.P. Pitaevskii, Statistical Physics, Part 2, Pergamon, Oxford (1980).

[16] Sanchayita Ghosh, Sutapa Ghosh and S. Chakrabary (in preparation). 
[17] Solid State Physics, N.W. Ashcroft and N.D. Mermin, Saunders College Publ. New York, (1976).

Acknowledgment: SC is thankful to Department of Science and Technology, Govt. of India, for partial support of this work, Sanction number:SP/S2/K3/97(PRU). 